\title{
A Cross Sectional Study of Comparison between Individual Height and Median Nerve Conduction Velocity
}

\author{
Dr. Dipti V. Thakker ${ }^{1}$, Dr. Viren B. Kariya ${ }^{2 *}$
}

${ }^{1}$ Associate Professor, Physiology Department, PDU Government Medical College, Rajkot (PhD student, Gujarat University), Gujarat, India
${ }^{2}$ Associate Professor, Anatomy Department, PDU Government Medical College, Rajkot, Gujarat, India

DOI: $10.36348 /$ sijap.2019.v02i11.004

| Received: 08.11.2019 | Accepted: 15.11.2019 | Published: 28.11.2019

*Corresponding author: Dr. Viren B. Kariya

\section{Abstract}

Introduction: There is negative correlation between conduction velocities in both the Median sensory and Ulnar nerves and an individual's height, which likely accounts for the fact that, among most of the adult population, for each inch increase in height conduction velocities between the wrist and digits of an individual's hand decrease by $0.5 \mathrm{~m} / \mathrm{s}$. Consequence of it results in, impulse latencies within the Median, Ulnar, and Sural nerves increases with height. There is negative correlation between height and the amplitude of impulses in the sensory nerves. Functions of peripheral nerves and their parameters can be assessed by Nerve conduction studies (NCS). These parameters are known to vary with anthropometric measurements. Objective: To study the effect of height on the NCS variables in the median nerves of the limbs. Material and method: The study was conducted in 196 healthy individuals. The anthropometric parameters like height, weight and BMI were calculated. The compound muscle action potential (CMAP) and the sensory nerve action potential (SNAP) were recorded. Statistical analysis: The correlation of height with the median NCS variables were analysed by using Z test for two samples. Results: After the adjustment of other anthropometric factors, height $(161.8673+8.140608)$ showed negative correlation with conduction velocities of median motor and sensory nerves of both the hands. Conclusion: Height showed a significant negative correlation with the median motor and sensory NCS parameters.

Keywords: Height, Nerve conduction study (NCS), Compound muscle action potential (CMAP), Sensory nerve action potential (SNAP), Median nerve, Motor nerve, Sensory nerve.

\footnotetext{
Copyright @ 2019: This is an open-access article distributed under the terms of the Creative Commons Attribution license which permits unrestricted use, distribution, and reproduction in any medium for non-commercial use (NonCommercial, or CC-BY-NC) provided the original author and source are credited.
}

\section{INTRODUCTION}

Electrical conduction of motor and sensory nerves of the human body is assessed by Nerve Conduction Velocity, which is a part of electrodiagnostic procedures, that help in establishing the type and nature of the nerve and commonly used to evaluate function of nerve. Nerve conduction velocity is affected by many physiological and technical variables. Physiological variables such as age, height, gender, upper limb versus lower limb, temperature affects conduction velocity. Diameter and myelination of the nerve fibres also affect nerve conduction velocity [1].

Nerve conduction studies (NCS) are performed to diagnose the disorders of the peripheral nervous system [2, 3]. These enable the clinicians to differentiate the two major groups of peripheral diseases: demyelination and axonal degeneration [4]. These also help in localizing the site of the lesions [5,6]. NCS consist primarily of the assessment of three types of nerves: motor, sensory and mixed. Motor NCS include the assessment of the compound muscle action potential (CMAP), whereas sensory NCS include the assessment of the sensory nerve action potentials (SNAP) of the accessible peripheral nerves in the upper and lower limbs. Commonly examined nerves are median, ulnar, radial, common peroneal, tibial and the sural nerves. The parameters of CMAP which are commonly measured include latency, amplitude, duration, conduction velocity and late response, e.g., Fwaves. Similarly, for SNAP, latency, amplitude and conduction velocity are routinely measured [7]. These parameters are known to vary with demographic profile, anthropometric measurements such as height, BMI, etc of the population which is studied and the laboratory conditions of the test1. Many studies have been done previously to evaluate the influence of the anthropometric factors such as age, height and body mass index on the nerve velocities [8]. However, a majority of these studies were based on the western 
population. Therefore, this study was designed to find the effect of height on the NCS parameters of the peripheral nerves in the upper and lower limbs among our healthy population.

\section{AIMS AND OBJECTIVES}

To study the correlation of height with the median motor and sensory $\mathrm{NCV}$ of the healthy individuals

\section{METHODS}

Cross - Sectional and Descriptive study was conducted in the Department of Physiology, B J Medical College, and Ahmedabad after obtaining clearance certificate from ethical committee. The study group included 196 healthy individuals of various age group. Subject with present history of fever, neurological abnormalities, any limb deformities and history of systemic diseases like diabetes mellitus, hypertension, were excluded. They were screened for any history of drugs/alcohol intake or medical illness which was likely to affect the nerve conduction study parameters on the basis of clinical history and physical examination, including a detailed neurological assessment. Informed consent was taken. The Anthropometric factors: age, sex, height, weight, body mass index and body surface were recorded.

Height and weight measured and BMI was calculated. The room temperature of the laboratory was maintained at the thermo neutral zone i.e. $26 \pm 2$ degree Celsius. Further, the subjects were made comfortable with the laboratory set up and conditions and were advised to relax completely during the recording. The CMAP and SNAP were recorded under standard laboratory conditions by using RMS EMG EPMK II, it is one of the latest software with facilities for nerve conduction velocity.

The Motor NCS variables: For each stimulation site, CMAP latency, amplitude, duration, conduction velocity and F-waves of the median, ulnar, radial, common peroneal and the tibial nerves were recorded.
The Sensory NCS variables: For each stimulation site, SNAP latency, duration, amplitude, and the conduction velocity of the median, ulnar, and radial and the sural nerves were recorded.

Nerve conduction velocity was calculated by formula and expressed in meter/second

Distance between stimulation sites ( $\mathrm{mm})$

Difference between latencies (ms)

\section{ETHICAL APPROVAL}

This study was approved by the ethical review committee of B J Medical College, Ahmedabad.

\section{STATISTICAL ANALYSIS}

The data which were collected were first entered into a Microsoft Excel sheet and were then statistically analysed by using the SPSS. Z test for two samples was applied to find the correlation of height with the NCS variables, while adjusting for other parameters. A significant difference was considered at $\mathrm{p}$ values which were less than 0.0 .

\section{RESULTS}

Cross - Sectional and Descriptive study was undertaken in 196 healthy individuals of different age groups. The mean (+SD) height for the subjects is $161.8673 \pm 8.140608$. Height was negatively associated with sensory and motor nerve conduction velocity of the median nerve $(\mathrm{P}<0.05)$. Age and height are the most important variables for median and ulnar distal sensory conduction velocities and latencies.

The mean height of the individuals is 161.8673 $+8.140608 \mathrm{~cm}$. Median Motor Nerve conduction velocity in right hand is 55.47082 \pm 5.349786 meter/second. Median Motor Nerve conduction velocity in left hand is $51.74587 \pm 4.989422$ meter/second. Median Sensory Nerve conduction velocity in right hand is 57.30128 \pm 5.356926 meter/second. Median Sensory Nerve conduction velocity in left hand is $52.77944 \pm 5.521057$ meter/second.

Table-1: Correlation between height and median motor nerve conduction velocity

\begin{tabular}{|l|l|l|}
\hline Median motor nerve conduction velocity (Right hand) & Mean: 55.47082 & p Value < 0.005 \\
\cline { 2 - 2 } Significant & SD: 5.349786 & \\
\hline Median motor nerve conduction velocity (Left hand) & Mean: 51.74587 & \\
\cline { 2 - 2 } & SD: 4.989422 & \\
\hline
\end{tabular}

There is negative correlation between height and median motor nerve conduction velocity of right and left hand (p value $<0.005$ ). 


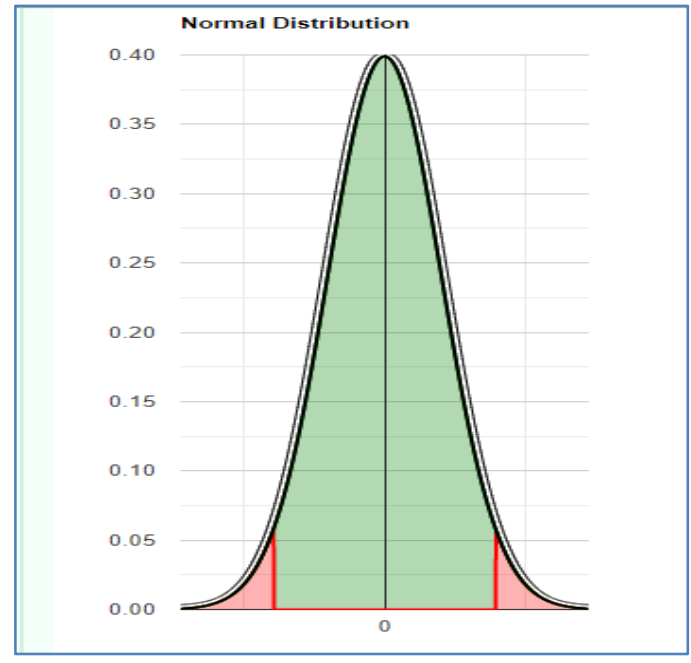

Median motor nerve conduction study (Right hand)

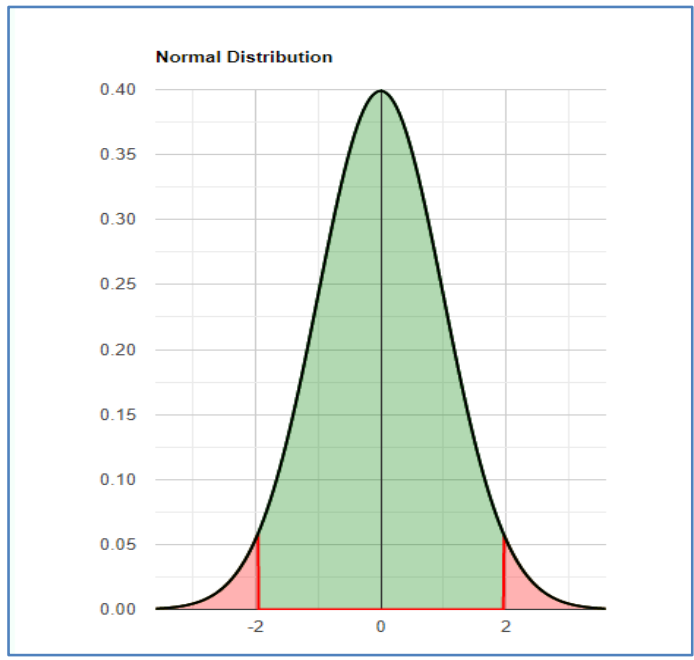

Median motor nerve conduction study (Left hand)

Table-2: Correlation between height and median Sensory nerve conduction velocity

\begin{tabular}{|c|c|c|}
\hline \multirow{2}{*}{ Median sensory nerve conduction velocity (Right hand) } & Mean: 57.30128 & \multirow{4}{*}{$\begin{array}{l}\mathrm{p} \text { Value }<0.005 \\
\text { Significant }\end{array}$} \\
\hline & SD:5.356926 & \\
\hline \multirow[t]{2}{*}{ Median sensory nerve conduction velocity (Left hand) } & Mean: 52.77944 & \\
\hline & SD:5.521057 & \\
\hline
\end{tabular}

There is negative correlation between Median sensory nerve conduction velocity of right and left hand ( $\mathrm{p}$ value $<0.005$ )

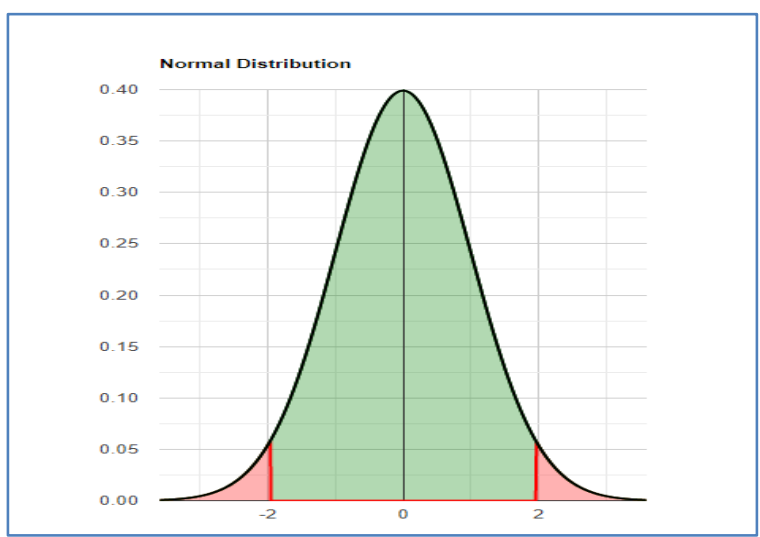

Median sensory nerve conduction velocity (Right hand)

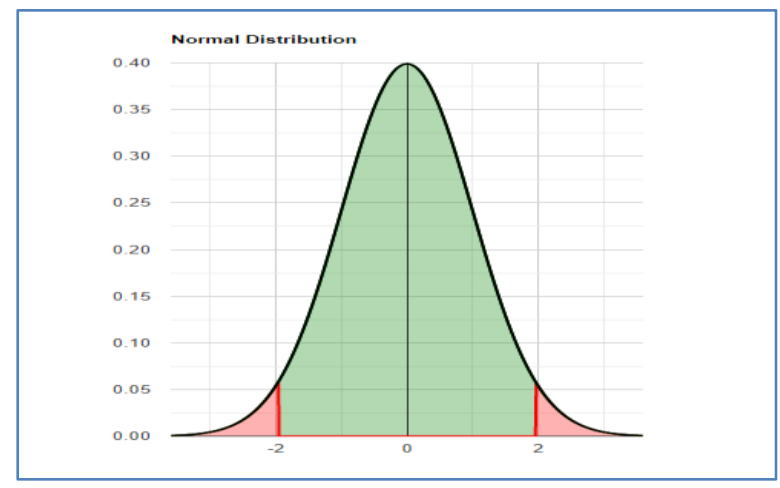

Median sensory nerve conduction study (Left hand)

\section{DISCUSSION}

The aim of our study is to explore the effect of height on the NCS variables in the healthy adults of our population. We found a negative correlation between height and the Nerve conduction velocity of median motor and sensory nerves of both hands [9]. There is negative correlation between the distal fibre diameter and height, which explains the decreased SNAP amplitude of the sural nerve. Hennessey WJ et al and Kokotis $\mathrm{P}$ et al. also reported height to be negatively associated with the sensory amplitude $[10,11]$.

Contrary to the reports of our study, Stetson DS found height to be negatively associated with the sensory amplitudes of the median and the ulnar nerves [9].

Distal axonal tapering in the nerves explains the effect. Even Campbell proposed that a decrease in diameter occurs abruptly at a given distance from the cell body [12].

Williams discovered that in mature rabbit nerves, the diameter of peripheral motor axon was about half that of ventral spinal nerve root fibres [13]. Height related slowing of nerve conduction velocity was observed in this study [14]. Clinical recognition of this height effect is important; otherwise an individual with mildly slowed peripheral nerve conduction velocity solely related to large stature may be labelled as abnormal.

Hennessey WJ et al. and Stetson DS et al. reported height to be positively associated with the sensory latencies $[9,10]$. S. Saeed et al. in their study, found an increase in the latency of the sural sensory nerve with increasing height [15]. 
Rivner $\mathrm{MH}$ et al. found that height was positively correlated with the latencies of the sural, peroneal, tibial, and the median nerves which were studied, which was similar to that which was found in our study [16]. It showed a negative correlation with the conduction velocities of the bilateral ulnar motor and the left median sensory nerves. Takano $\mathrm{K}$ et al. and Gutjahr L et al. supported the possibility of an inverse correlation of the conduction velocity of the ulnar nerve with height [17].

Logically, taller subjects have a longer conduction time of late response, because of a longer conduction distance. Peioglou HS et al. and Lin KP et al. found a strong correlation between the $\mathrm{F}$ latencies and height $[18,19]$. Likewise, in the study which was done by Puksa $\mathrm{L}$ et al. and Toyokura $\mathrm{M}$ et al. the minimal latency of the F-wave study was found to increase with height in studies on the upper and lower limbs [20, 21].

In summary, this study shows that height influences the nerve conduction study parameters of both the motor and the sensory nerves. From the nerve conduction data, diagnostic conclusions which are made without making corrections for height may be invalid in patients who are taller and shorter than average individuals. Our study has many similarities and some dissimilarity with the earlier reported NCS variables. The probable reasons could be the true difference among the populations and the small sample size. More strength to the results can be added by a larger sample size. Nevertheless, this study may be used as a preliminary working reference while reporting the clinical NCS findings and for further research in this field. In this way, this study has a place of significance.

In mature rabbit nerves, Williams [13] found that peripheral motor axon diameter was about half that of ventral spinal nerve root fibres and, despite an increase in myelin sheath thickness, there was an overall decrease in total fibre diameter. Our results, which showed an association with median sensory distal conduction velocities, are consistent with the contention that tapering occurs distal to the wrist. The association between height and ulnar, but not median, midpalm-wrist sensory measures may have an anatomical basis, e.g., more proximal tapering in the ulnar than median nerve, or may be an artefact.

\section{CONCLUSION}

Height showed a significant negative correlation with the nerve conduction parameters of most of the motor nerves and a few sensory nerves. Diagnostic conclusions which were made from the nerve conduction data without making corrections for height may be invalid in patients who are taller and shorter than average individuals. These values must also be considered while developing standard/reference normative data for different nerves.

\section{REFERENCES}

1. Misra, U. K., \& Kalita, J. (2019). Clinical neurophysiology: nerve conduction, electromyography, evoked potentials. Elsevier Health Sciences.

2. Preston, D. C., \& Shapiro, B. E. (2012). Electromyography and Neuromuscular Disorders E-Book: Clinical-Electrophysiologic Correlations (Expert Consult-Online and Print). Elsevier Health Sciences.

3. Misulis, K. E., \& Head, T. C. (2003). Nerve conduction study and electromyography. Essentials of Clinical Neurophysiology". 3rd Ed. Burlington: Butterworth-Heinemann, 129-144.

4. Kouyoumdjian, J. A., Zanetta, D. M., \& Morita, M. P. (2002). Evaluation of age, body mass index, and wrist index as risk factors for carpal tunnel syndrome severity. Muscle \& Nerve: Official Journal of the American Association of Electrodiagnostic Medicine, 25(1), 93-97.

5. Evans, B. A., \& Daube, J. R. (1984, January). A comparison of 3 electrodiagnostic methods of diagnosing carpal-tunnel syndrome. In muscle \& nerve (vol. 7, no. 7, pp. 565-565). 605 third ave, new york, ny 10158-0012: john wiley \& sons inc.

6. Stevens, J. C. (1997). AAEM minimonograph\# 26: the electrodiagnosis of carpal tunnel syndrome. Muscle \& Nerve: Official Journal of the American Association of Electrodiagnostic Medicine, 20(12), 1477-1486.

7. Misulis, K. E., \& Head, T. C. (2003). Nerve conduction study and electromyography. Essentials of Clinical Neurophysiology". 3rd Ed. Burlington: Butterworth-Heinemann, 129-144.

8. Trojaborg, W. T., Moon, A., Andersen, B. B., \& Trojaborg, N. S. (1992). Sural nerve conduction parameters in normal subjects related to age, gender, temperature, and height: a reappraisal. Muscle \& Nerve: Official Journal of the American Association of Electrodiagnostic Medicine, 15(6), 666-671.

9. Stetson, D. S., Albers, J. W., Silverstein, B. A., \& Wolfe, R. A. (1992). Effects of age, sex, and anthropometric factors on nerve conduction measures. Muscle \& Nerve: Official Journal of the American Association of Electrodiagnostic Medicine, 15(10), 1095-1104.

10. Hennessey, W. J., Falco, F. J., Goldberg, G., \& Braddom, R. L. (1994). Gender and arm length: influence on nerve conduction parameters in the upper limb. Archives of physical medicine and rehabilitation, 75(3), 265-269.

11. Kokotis, P., Mandellos, D., Papagianni, A., \& Karandreas, N. (2010). Nomogram for determining lower limit of the sural response. Clinical Neurophysiology, 121(4), 561-563.

12. Campbell Jr, W. W., Ward, L. C., \& Swift, T. R. (1981). Nerve conduction velocity varies inversely with height. Muscle \& Nerve: Official Journal of 
the American Association of Electrodiagnostic Medicine, 4(6), 520-523.

13. Williams, P. L., \& Wendell-Smith, C. P. (1971). Some additional parametric variations between peripheral nerve fibre populations. Journal of anatomy, 109(Pt 3), 505.

14. Rivner, M. H., Swift, T. R., \& Malik, K. (2001). Influence of age and height on nerve conduction. Muscle \& nerve, 24(9), 1134-1141.

15. Saeed, S., \& Akram, M. (2008). Impact of anthropometric measures on sural nerve conduction in healthy subjects. $J$ Ayub Med Coll Abbottabad, 20(4), 112.

16. Rivener, M. H., Swift, T. R., Crout, B. O., \& Rhodes, K. P. (1990). Toward more rational nerve conduction interpreations: The effect of height. Muscle \& Nerve: Official Journal of the American Association of Electrodiagnostic Medicine, 13(3), 232-239.

17. Takano, K., Kirchner, F., Steinicke, F., Langer, A., Yasui, H., \& Naito, J. (1991). Relation between height and the maximum conduction velocity of the ulnar motor nerve in human subjects. The Japanese journal of physiology, 41(3), 385-396.

18. Peioglou-Harmoussi, S., Howel, D., Fawcett, P. R., \& Barwick, D. D. (1985). F-response behaviour in a control population. Journal of Neurology, Neurosurgery \& Psychiatry, 48(11), 1152-1158.

19. Lin, K. P., Chan, M. H., \& Wu, Z. A. (1993). Nerve conduction studies in healthy Chinese: correlation with age, sex, and height and skin temperature. Zhonghua yi xue za zhi= Chinese medical journal; Free China ed, 52(5), 293-297.

20. Puksa, L., Stålberg, E., \& Falck, B. (2003). Reference values of $\mathrm{F}$ wave parameters in healthy subjects. Clinical Neurophysiology, 114(6), 10791090 .

21. Toyokura, M., \& Ishida, A. (2000). Diagnostic sensitivity of predicted F-wave latency by age, height, and MCV.Acta neurologica scandinavica, 102(2), 106-113. 